\title{
Effect of mild baking on superconducting niobium cavities investigated by sequential nanoremoval
}

\author{
A. Romanenko, ${ }^{*}$ A. Grassellino, and F. Barkov \\ Fermi National Accelerator Laboratory, Batavia, Illinois 60510, USA \\ J. P. Ozelis \\ Facility for Rare Isotope Beams, East Lansing, Michigan 48824, USA \\ (Received 23 August 2012; published 7 January 2013)
}

\begin{abstract}
The near-surface nanostructure of niobium determines the performance of superconducting microwave cavities. Subtle variations in surface nanostructure lead to yet unexplained phenomena such as the dependence of the quality factor of these resonating structures on the magnitude of rf fields-an effect known as the " $Q$ slopes". Understanding and controlling the $Q$ slopes is of great practical importance for particle accelerators. Here we investigate the mild baking effect $-120^{\circ} \mathrm{C}$ vacuum baking for 48 hours-which strongly affects the $Q$ slopes. We used a hydrofluoric acid rinse alternating with oxidation in water as a tool for stepwise material removal of about 2 nanometers/step from the surface of superconducting niobium cavities. Applying removal cycles on mild baked cavities and measuring the quality factor dependence on the rf fields after one or several such cycles allowed us to explore the distribution of lossy layers within the first several tens of nanometers from the surface. We found that a single HF rinse results in the increase of the cavity quality factor. The low field $Q$ slope was shown to be mostly controlled by the material structure within the first six nanometers from the surface. The medium field $Q$ slope evolution was fitted using linear $(\propto B$ peak surface magnetic field) and quadratic $\left(\propto B^{2}\right)$ terms in the surface resistance and it was found that best fits do not require the quadratic term. We found that about 10 nanometers of material removal are required to bring back the high field $Q$ slope and about 20-50 nanometers to restore the onset field to the prebaking value.
\end{abstract}

DOI: 10.1103/PhysRevSTAB.16.012001

PACS numbers: 74.25.nn, 68.35.Dv, 81.40.-z

\section{INTRODUCTION}

The quality factor of superconducting niobium cavities exhibits a field dependence characterized by three distinct regions in the $Q_{0}\left(B_{\text {peak }}\right)$ curve (see Ref. [1] for review). Underlying mechanisms, which govern these so-called $Q$ slopes, are not clear despite a number of studies in the recent decade. One of the major obstacles is the absence of a full nanoscale understanding of the material changes in the magnetic field penetration depth $(\lessgtr 100 \mathrm{~nm})$ brought about by different treatments applied on cavities. Such treatments include electropolishing (EP), buffered chemical polishing (BCP), $120^{\circ} \mathrm{C}$ baking, and $600-800^{\circ} \mathrm{C}$ baking in vacuum furnaces.

While there are no established methods to control low and medium field $Q$ slopes, it was discovered that the high field $Q$ slope (HFQS) can be removed by a so-called mild baking - an in situ ultrahigh vacuum annealing of cavities at $90-145^{\circ} \mathrm{C}$ for the duration of $12-48$ hours [2].

\footnotetext{
*aroman@fnal.gov

Published by the American Physical Society under the terms of the Creative Commons Attribution 3.0 License. Further distribution of this work must maintain attribution to the author(s) and the published article's title, journal citation, and DOI.
}

In addition to the removal of the HFQS, mild baking was demonstrated to lead to a decrease in BCS surface resistance by up to $\sim 50 \%$ and to an increase in the residual resistance. The mechanism which underlies the mild baking effect remains the main unresolved issue. Several important clues were obtained by experiments on cavities. First, by oxipolishing experiments the change in BCS surface resistance after $145^{\circ} \mathrm{C} 45$ hours bake was found to extend down to about 300 nanometers from the surface [3]. Second, it was demonstrated by cavity anodizing experiments [4,5] that modifications introduced by mild baking at $100-120^{\circ} \mathrm{C}$, which are affecting the high field $Q$ slope, are confined to only $\sim 20-30$ nanometers from the surface. It was also shown that vacuum conditions for mild baking may not be crucial and Ar or even atmospheric air can be used [6].

While anodizing studies provided an indication of the crucial length scales for the mild baking effect on the high field $Q$ slope, it is important to study in detail how all of the $Q$ slopes (and not only HFQS) evolve when bakingmodified material is being removed. Such detailed information is important for understanding how surface nanostructure leads to the particular low, medium, and high field $Q$ slopes and to provide experimental data for models to compare against. Furthermore, the anodizing 


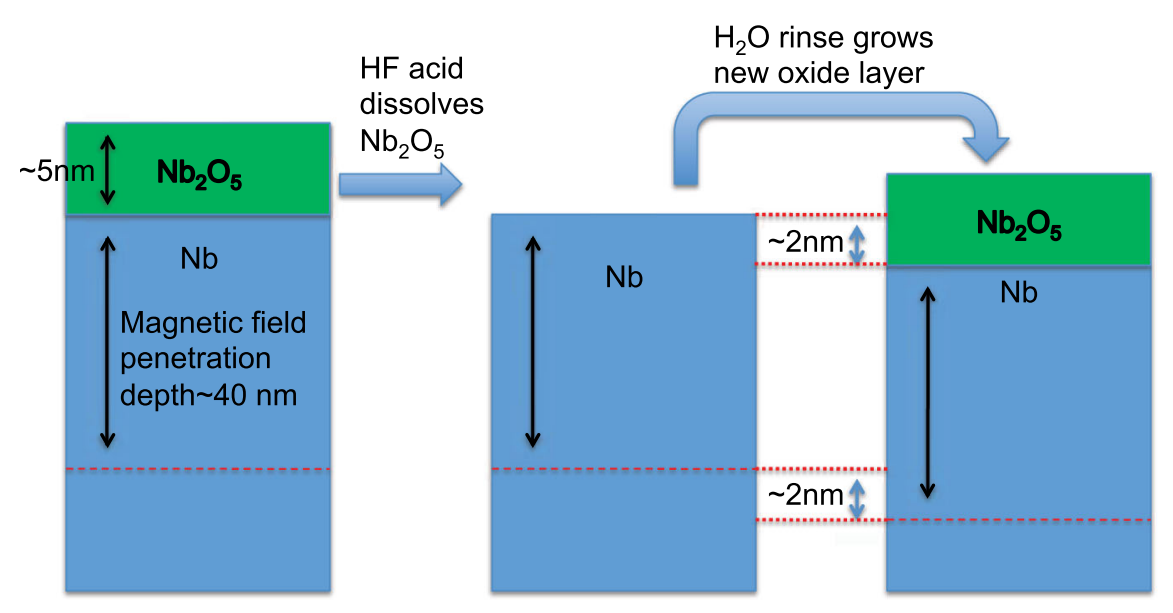

FIG. 1. Schematic of the nanoremoval during a single HF/water rinse cycle.

process involves an electric potential and oxygen diffusion, which potentially may play a role in the observed changes. Thus, using a different method of nanoremoval serves as an independent cross-check of the anodizing results.

In this article we report measurements on the cavities where, as a means of nanoremoval, we utilized a hydrofluoric acid (48\% concentrated) rinse for 5 minutes followed by several ultrapure water rinses. Since HF is the acid attacking niobium pentoxide [7], HF is typically used in superconducting radio frequency (SRF) technology as a solution component in standard chemical treatments such as BCP or EP. It is also used in water solution to, e.g., remove a thick oxide formed by anodizing, during oxipolishing. The oxide layer is then regrown upon air or water exposure within minutes [8]. Thus, we assume the following sequence in our experiments: (1) HF rinse removes the niobium pentoxide $\left(\mathrm{Nb}_{2} \mathrm{O}_{5}\right)$ layer; (2) air exposure/ water rinse regrows a new $\mathrm{Nb}_{2} \mathrm{O}_{5}$ layer of about 4-5 nanometers equilibrium thickness [9]. The oxide thickness of 4-5 nanometers was also directly confirmed by TEM investigations on electropolished cavity cutouts. Because of the water-based HF solution, it is hypothetically possible that some etching may be going on. If it is nonnegligible over the time of each rinse $(5 \mathrm{~min})$ then all of our thickness estimates should be taken as lower bounds. As it will be shown below, our length scales obtained neglecting etching agree reasonably well with anodizing experiments and, hence, our simple model is adequate.
Forming 4-5 nanometers of pentoxide consumes about 1.5-2 nanometers of niobium as estimated from the corresponding densities. Hence, the net effect of a single HF rinse cycle is to regrow a new wet oxide and push the layer with rf currents deeper by about 1.5-2 nanometers. A schematic sequence of events during such treatment is shown in Fig. 1. The primary difference of such a process from anodizing is the absence of electric potential. A possible side effect of HF rinsing (as well as oxipolishing used in Ref. [3]) may be due to the entering of hydrogen into niobium whenever the protective $\mathrm{Nb}_{2} \mathrm{O}_{5}$ layer is not present. Some previous studies [10] addressed this issue but detailed understanding is not yet developed.

We report $\mathrm{rf}$ measurements of the quality factor versus temperature after nanoremoval steps, from which we extract how superconducting parameters change as a function of depth, and $Q_{0}\left(B_{\text {peak }}\right)$ curves, which provide information on the change in $Q$ slopes.

\section{EXPERIMENTAL}

Three different $1.3 \mathrm{GHz}$ niobium cavities of TESLA [11] elliptical shape were used for these studies. Niobium properties and surface treatments that were applied on cavities are summarized in Table I.

Before the series of HF rinses each of the cavities was baked at $120^{\circ} \mathrm{C}$ for 48 hours in vacuum. As expected, it removed the high field $Q$ slope in EP cavities TE1ACC002

TABLE I. List of cavities used for experiments.

\begin{tabular}{lccc}
\hline \hline Cavity ID & Material & Manufacturer & Treatment \\
\hline TE1ACC002 & RRR $\gtrsim 200$, grain size $\sim 50 \mu \mathrm{m}$ & ACCEL & Bulk EP $(120 \mu \mathrm{m})+$ tumbling + light EP $(20 \mu \mathrm{m})$ \\
TE1ACC005 & RRR $\gtrsim 200$, grain size $\sim 50 \mu \mathrm{m}$ & ACCEL & Bulk EP $(120 \mu \mathrm{m})$ \\
TE1AES003 & RRR $\gtrsim 200$, grain size $\sim 50 \mu \mathrm{m}$ & AES & Bulk BCP $(120 \mu \mathrm{m})$ \\
\hline \hline
\end{tabular}




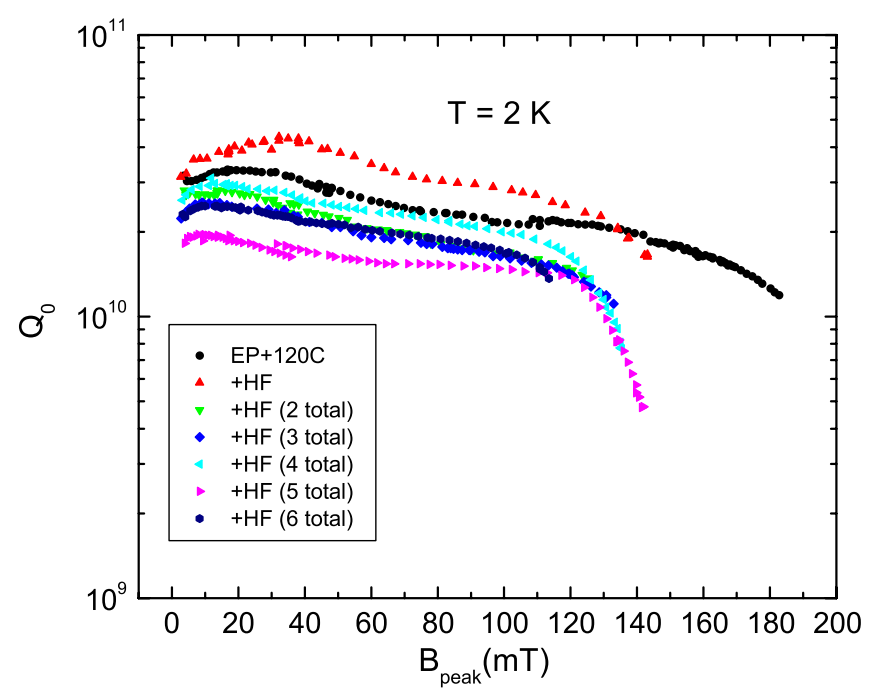

FIG. 2. Cavity rf test results after multiple HF rinse cycles for the electropolished tumbled cavity TE1ACC002.

and TE1ACC005 while only shifting it to slightly higher fields in the BCP cavity TE1AES003.

After each rinsing cycle(s) we measured the cavity quality factor $Q_{0}$ at $2 \mathrm{~K}$ as a function of the accelerating gradient $E_{\text {acc }}$. In some tests additional measurements of $Q_{0}(T)$ at $E_{\text {acc }}=5 \mathrm{MV} / \mathrm{m}$ in the temperature range $1.5<$ $T<2 \mathrm{~K}$ were performed. For TESLA elliptical geometry the ratio of peak surface magnetic field $B_{\text {peak }}$ to $E_{\text {acc }}$ is $4.26 \mathrm{mT} /(\mathrm{MV} / \mathrm{m})$ and for convenience all test results are plotted as $Q_{0}\left(B_{\text {peak }}\right)$. All rf measurements at $2 \mathrm{~K}$ for the three different cavities we used are presented in Figs. 2-4. For all measurements at $2 \mathrm{~K}$, the estimated error in $Q_{0}$ is about $10 \%$, while $B_{\text {peak }}$ has about $5 \%-7 \%$ error. In all tests

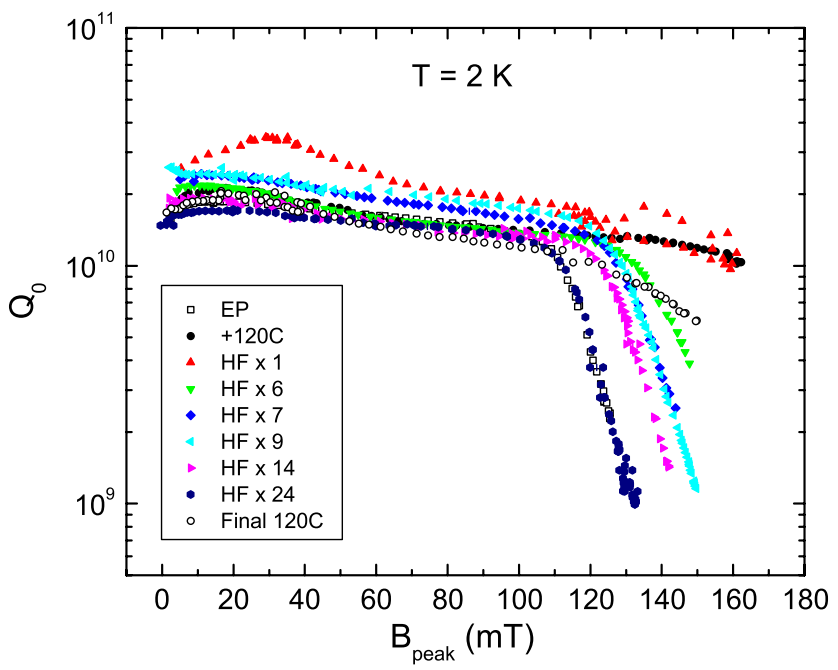

FIG. 3. Cavity rf test results after multiple HF rinse cycles for the electropolished cavity TE1ACC005. No field emission was present except for the final $120^{\circ} \mathrm{C}$ test.

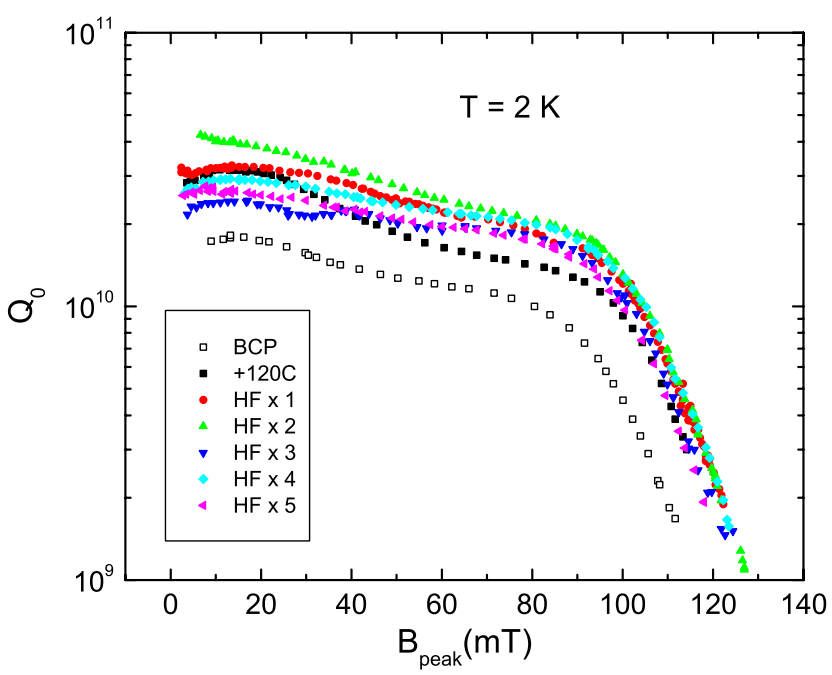

FIG. 4. Cavity rf test results after multiple HF rinse cycles for the buffered chemical polished cavity TE1AES003.

highest $B_{\text {peak }}$ was limited by either quench or available rf power.

To visualize better the evolution of $Q_{0}\left(B_{\text {peak }}\right)$ curves, plots of $Q_{0} / Q_{\text {max }}\left(B_{\text {peak }}\right)$, where $Q_{\max }$ is the maximum quality factor in each case, are shown in Fig. 5.

\section{DISCUSSION}

\section{A. Evolution of residual and BCS resistances}

It is important to understand how effective values of the residual and BCS surface resistances, superconducting gap, and electron mean-free path vary in mild baking cavities with distance from the surface. We can semiquantitatively extract such values from fits to the temperature dependence of the quality factor $Q_{0}(T)$ measured after each HF rinse cycle. This information can help in understanding the underlying driving factors for the mild baking effect and $Q$ slopes at the material level.

We have performed such measurements only on the electropolished tumbled cavity TE1ACC002 due to the technical constraints. In future experiments we plan to repeat $Q(T)$ measurements on other cavities prepared similarly to TE1ACC002. For TE1ACC002 we measured the temperature dependence $Q_{0}(T)$ at $E_{\text {acc }}=5 \mathrm{MV} / \mathrm{m}$ $\left(B_{\text {peak }} \approx 21 \mathrm{mT}\right)$ for $1.55<T<2 \mathrm{~K}$ in all rf tests in addition to the $2 \mathrm{~K} Q$ curve. The average surface resistance calculated as $\bar{R}_{s}=G / Q_{0}$, where $G=270$ is a geometry factor, is shown in Fig. 6 for data obtained after each of the HF rinses.

It is worth mentioning that, since a fixed input coupler with the external quality factor of $Q_{\mathrm{ext}} \sim 10^{10}$ was used in all rf tests, measurement errors are getting large $\left(\Delta Q / Q_{0} \gtrsim 50 \%\right)$ as the $Q_{0}$ increases beyond $10^{11}$ making precise $Q_{0}$ measurement a challenge. Thus, most of the results at lower temperatures with very high quality factors 

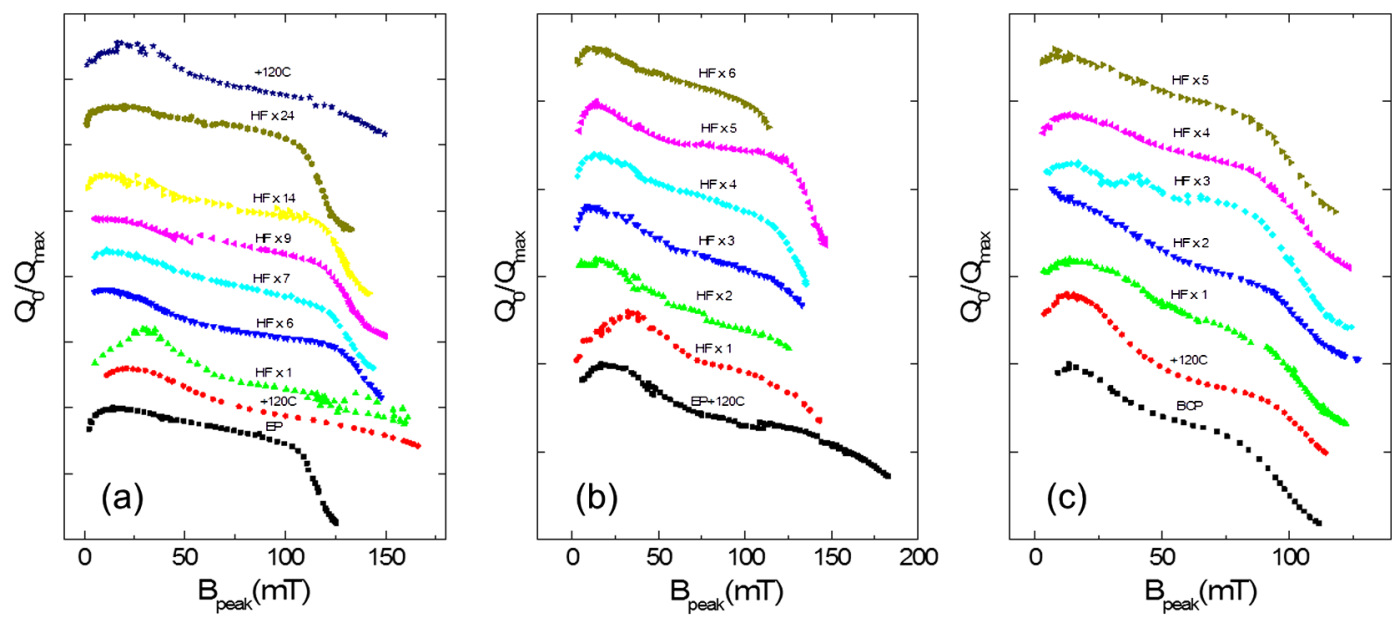

FIG. 5. Evolution of $Q_{0}\left(B_{\text {peak }}\right)$ curve shapes with multiple HF rinse cycles for: (a) electropolished cavity TE1ACC005; (b) electropolished cavity TE1ACC002 with tumbling to a mirror smooth finish as one of the processing steps; (c) buffered chemical polished cavity TE1AES003.

should be taken with caution providing a qualitative performance comparison rather than exact quantitative one.

We used the code [12] based on the original Halbritter's program [13] for the weakly coupled BCS model with diffuse scattering to fit $R_{s}(T)$ with fixed parameters $T_{c}=$ $9.25 \mathrm{~K}, \lambda_{0}=30 \mathrm{~nm}, \xi_{0}=39 \mathrm{~nm}$, and varying free parameters-residual resistance $R_{\text {res }}$, superconducting gap $\Delta /\left(k_{B} T_{c}\right)$, and electron mean-free path $l_{\mathrm{mfp}}$. The value of $\lambda_{0}$ was selected by performing a global fit on all curves with the optimal value $(30 \mathrm{~nm})$ close to the one reported in recent $\mu$ SR investigations on cavity cutouts [14].

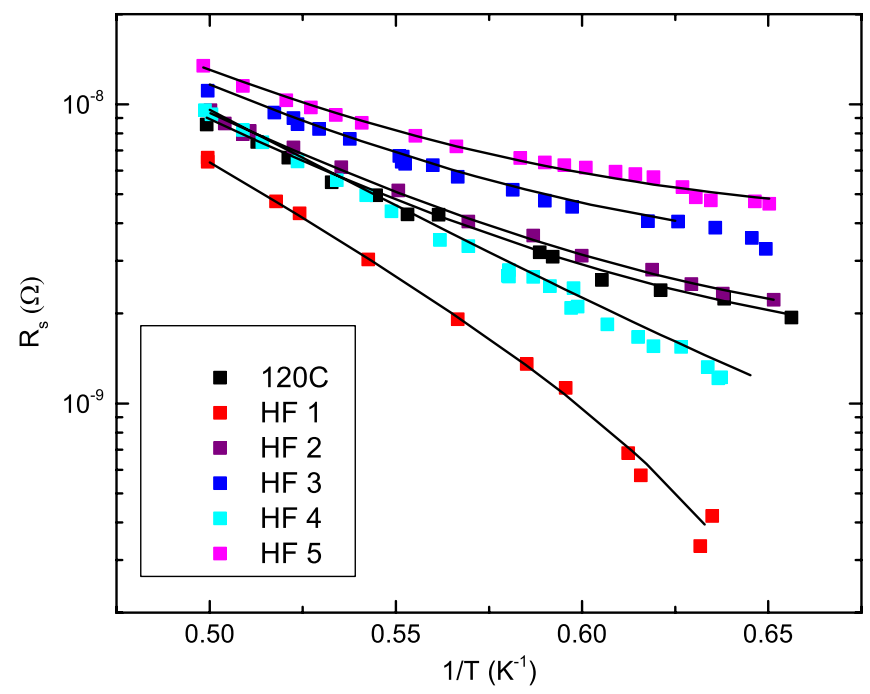

FIG. 6. Average surface resistance $R_{s}=G / Q_{0}$ at $H_{\text {peak }} \approx$ $21 \mathrm{mT}$ after multiple HF rinse cycles for the electropolished tumbled cavity TE1ACC002. Relative errors $\Delta R_{s} / R_{s}$ are estimated at about $10 \%$ at $2 \mathrm{~K}$ and $50 \%$ at lowest temperatures $(\sim 1.6 \mathrm{~K})$. Black lines represent fits to the data based on the weakly coupled BCS approximation using the code from [12].
Fit results are summarized in Table II. It should be noted that BCS surface resistance is only weakly dependent on the electron mean-free path within the temperature range we studied. As a consequence, our extracted values of $l_{\mathrm{mfp}}$ suffer from a big uncertainty prohibiting any definitive conclusions regarding its evolution with HF rinses. On the other hand, the residual resistance can be extracted pretty accurately and, hence, both $R_{\text {res }}$ and $R_{\mathrm{BCS}}$ are more reliable indicators of the changes.

We should comment on the negative residual resistance after a single HF rinse. Since the quality factor is very high $\left(>4 \times 10^{10}\right)$ even at $2 \mathrm{~K}$ and increasing rapidly upon cooldown, the error is large for most of the points in the $Q_{0}(T)$ curve. Thus, the negative residual resistance may be an artifact reflecting the low residual resistance value affected by the measurement error.

Our data show a gradual increase in BCS surface resistance with the depth of the material removed starting from the second HF rinse. Such an increase is consistent with the observations of Kneisel [3] and supports a gradual consumption of the lower mean-free path layer by HF rinsing.

TABLE II. Changes in the superconducting properties after HF rinsing cycles for the bulk EP tumbled cavity TE1ACC002. Shown errors include standard deviation of the fit only.

\begin{tabular}{lccc}
\hline \hline Treatment & $R_{\text {res }}(n \Omega)$ & $R_{\mathrm{BCS}}(\mathrm{n} \Omega)$ & $\Delta /\left(k T_{c}\right)$ \\
\hline $\mathrm{EP}+120^{\circ} \mathrm{C}$ & $1.3 \pm 0.2$ & 7.2 & $1.84 \pm 0.15$ \\
$\mathrm{HF}$ rinse $\times 1$ & $-0.4 \pm 0.1^{\mathrm{a}}$ & 7.4 & $1.86 \pm 0.01$ \\
$\mathrm{HF}$ rinse $\times 2$ & $1.5 \pm 0.2$ & 8.1 & $1.83 \pm 0.30$ \\
$\mathrm{HF}$ rinse $\times 3$ & $2.8 \pm 0.6$ & 8.3 & $1.81 \pm 0.53$ \\
$\mathrm{HF}$ rinse $\times 4$ & $0.2 \pm 0.1$ & 9.3 & $1.80 \pm 0.09$ \\
$\mathrm{HF}$ rinse $\times 5$ & $3.9 \pm 0.4$ & 9.5 & $1.80 \pm 1.6$ \\
\hline \hline
\end{tabular}

${ }^{a}$ Extracted as a fit parameter with the corresponding standard deviation. 
The residual resistance is decreased after a single HF rinse, which is followed by the increasing trend with subsequent rinses except for a rinse \#4. Thus, no systematic trend can be clearly observed after second and subsequent rinses.

\section{B. Single HF rinse as a way of $Q_{0}$ maximization}

One of the apparent effects observed on all three cavities is a significant increase in the quality factor at low and medium fields after a single $\mathrm{HF}$ rinse cycle. At $70 \mathrm{mT}$ the improvement in $Q_{0}$ is $16 \%$ (TE1ACC005), $31 \%$ (TE1ACC002), and 37\% (TE1AES003). It is interesting to mention that, for the bulk BCP cavity TE1AES003, the second HF rinse leads to yet further improvement in $Q_{0}$ with the total increase of $49 \%$. As mentioned above, mild baking has two side effects: a decrease in the BCS surface resistance $R_{\mathrm{BCS}}$ by a factor of up to 2, and an increase in the residual resistance $R_{\text {res }}$. Reversing an increase in $R_{\text {res }}$ and keeping the benefit in $R_{\mathrm{BCS}}$ should allow minimizing the total surface resistance. We believe the observed $Q_{0}$ increase may stem from the decrease of the residual resistance by a single HF rinse consistent with earlier literature reports [5]. This means that the increase in $R_{\text {res }}$ caused by mild baking is due to the changes in either the oxide or in the first 1.5-2 nm of niobium underneath it. If it is due to oxide changes, then it may be attributed to the formation of normal conducting $\mathrm{NbO}_{x}$ layer or clusters. If it is due to niobium underneath it may be caused by the interstitial oxygen enrichment.

It is worth noting that a very high quality factor $Q_{0}>2 \times 10^{11}$ at $1.6 \mathrm{~K}$ at low fields was measured in TE1ACC002 after the first HF rinse. This corresponds to a total surface resistance of the order of $1 \mathrm{nOhm}$. But due to the large errors caused by a fixed coupler at high $Q_{0}>10^{11}$ values described above, this result should be taken with caution.

Many future accelerators are based on the superconducting $\mathrm{rf}$ technology and are intended to be operated in a cw regime. It makes the minimization of the $\mathrm{rf}$ losses at a moderate field level (e.g. $70 \mathrm{mT}$ for Project X) a task of major importance since it directly translates into the costs associated with the required refrigeration power. Based on our findings, a single $\mathrm{HF}$ rinse performed after $120^{\circ} \mathrm{C}$ baking represents a simple technique to maximize low/ medium field $Q_{0}$ with minimal modifications to the existing processing sequence.

\section{C. $Q$ slopes}

One of the main goals of our study is to get insight into what is responsible for different $Q$ slopes. In particular, one of the questions is where the particular features/layers leading to the low, medium and high field $Q$ slopes are localized with respect to the niobium surface. To answer this question we analyze in detail the evolution of each of the $Q$ slopes with HF treatments and extract possible depth distributions of the corresponding parameters.

\section{Low field $Q$ slope}

Low field $Q$ slope (LFQS) is typically observed as an increase in the cavity quality factor $Q_{0}$ with the field in the range of surface magnetic fields of $0-20 \mathrm{mT}$. There is a very limited number of studies of this effect $[15,16]$ reported in the literature.

Several models have been put forward to explain the LFQS. One of the models is based on the presence of $\mathrm{NbO}_{x}$ clusters at the oxide-metal interface [17]. Another model relies on the presence of small weakly superconducting defects [18]. These models predict the inverse quadratic dependence of the surface resistance on the peak magnetic field (although due to different mechanisms):

$$
R_{s}=\frac{a}{H^{2}}+b .
$$

One more model is based on the hypothesis that the niobium surface in a cavity can be treated as a two-layer superconductor with the "dirty" superconductor film on top of bulk niobium [19].

Low field $Q$ slope can be easily explained by additional field-independent dissipated power. In fact, any dissipative mechanism which leads to a dissipated power $P_{\text {diss }}$ per unit area with the field dependence weaker than $\propto H^{2}$ will result in the low field $Q$ slope. Indeed, suppose we have additional losses $P^{*}(H)$. Then

$$
\begin{aligned}
Q_{0}(H) & =\frac{\omega U}{P_{\mathrm{diss}}} \propto \frac{\int_{V} H^{2} d v}{\int_{S} R_{s}(H) H^{2} d s+P^{*}(H)} \\
& \propto \frac{\mathrm{const}}{\mathrm{const}+P^{*}(H) / H^{2}} \Rightarrow R_{s}(H) \propto 1 / Q_{0}(H) \\
& =\mathrm{const}+\mathrm{const} \cdot P^{*}(H) / H^{2}
\end{aligned}
$$

which will be an increasing function of $H$ if $P^{*}(H)$ has a weaker than $H^{2}$ character, leading to the low field $Q$ slope. In particular, if $P^{*}(H)=$ const then we recover a LFQS parametrization as in Eq. (1).

We find that Eq. (1) describes our data reasonably well with several important exceptions. This finding points toward the constant with field additional dissipated power behind the LFQS for most of the cases as explained above.

The first exception is that LFQS in both TE1ACC002 and TE1ACC005 cavities after a single HF rinse could not be fitted with Eq. (1). The second exception is the absence of the low field $Q$ slope in both TE1ACC002 and TE1AES003 after two HF rinses. We have no explanation for these facts at the moment.

In Table III the values of the best fit parameters to our data based on Eq. (1) are presented. Corresponding plots of the best fit parameters as a function of approximate 
TABLE III. Parameters for the fits based on Eq. (1) of the low field $Q$ slope in the bulk electropolished (EP) cavity (TE1ACC005), bulk EP + tumbling + light EP cavity (TE1ACC002), and a buffered chemically polished (BCP) cavity (TE1AES003).

\begin{tabular}{|c|c|c|c|}
\hline TE1ACC005 & $a\left[10^{-10} \Omega(\mathrm{MV} / \mathrm{m})^{2}\right]$ & $b(\mathrm{n} \Omega)$ & $r^{2}$ \\
\hline EP not baked & 56.0 & 14.3 & 0.998 \\
\hline $\mathrm{EP}+120^{\circ} \mathrm{C}$ & 55.7 & 10.0 & 0.995 \\
\hline \multicolumn{4}{|l|}{$\mathrm{HF}$ rinse $\times 1^{\mathrm{a}}$} \\
\hline HF rinse $\times 6$ & 4.2 & 12.3 & 0.926 \\
\hline $\mathrm{HF}$ rinse $\times 7$ & 7.3 & 11.1 & 0.745 \\
\hline \multicolumn{4}{|l|}{ HF rinse $\times 9^{b}$} \\
\hline HF rinse $\times 14$ & 2.8 & 14.1 & 0.954 \\
\hline HF rinse $\times 24$ & 1.5 & 16.3 & 0.918 \\
\hline \multicolumn{4}{|l|}{ TE1ACC002 } \\
\hline $\mathrm{EP}+120^{\circ} \mathrm{C}$ & 27.3 & 8.0 & 0.934 \\
\hline \multicolumn{4}{|l|}{ HF rinse $\times 1^{a}$} \\
\hline \multicolumn{4}{|l|}{$\mathrm{HF}$ rinse $\times 2^{\mathrm{b}}$} \\
\hline $\mathrm{HF}$ rinse $\times 3$ & 6.5 & 10.7 & 0.913 \\
\hline HF rinse $\times 4$ & 8.0 & 9.1 & 0.974 \\
\hline HF rinse $\times 5$ & 25.2 & 13.1 & 0.974 \\
\hline HF rinse $\times 6$ & 11.1 & 10.7 & 0.997 \\
\hline \multicolumn{4}{|l|}{ TE1AES003 } \\
\hline BCP no bake & 34.5 & 14.8 & 0.977 \\
\hline \multicolumn{4}{|l|}{$\mathrm{BCP}+120^{\circ} \mathrm{C}^{\mathrm{a}}$} \\
\hline HF rinse $\times 1$ & 8.1 & 8.3 & 0.977 \\
\hline \multicolumn{4}{|l|}{ HF rinse $\times 2^{b}$} \\
\hline $\mathrm{HF}$ rinse $\times 3$ & 9.5 & 11.1 & 0.904 \\
\hline $\mathrm{HF}$ rinse $\times 4$ & 6.9 & 9.2 & 0.979 \\
\hline $\mathrm{HF}$ rinse $\times 5^{\mathrm{c}}$ & & & \\
\hline
\end{tabular}

${ }^{\mathrm{a}}$ Fit by Eq. (1) was not possible — a different field dependence is present.

${ }^{\mathrm{b}}$ No low field $Q$ slope was observed.

${ }^{\mathrm{c}}$ Too few points measured in the LFQS range.

thickness of niobium consumed by HF rinsing cycles are shown in Fig. 7.

LFQS "strength" characterized by $a$ starts at a high value after $120^{\circ} \mathrm{C}$ treatment. It is suppressed by the next two HF rinses with the values appearing to remain at approximately the same average value after third and subsequent $\mathrm{HF}$ rinses except for one outlier point at $\mathrm{HF} \times 5$ for TE1ACC002. Therefore, the low field $Q$ slope appears to be the very surface effect governed by the material within three HF rinses corresponding to about 4.5-6 nanometers from the surface.

The constant $b$, which captures the variation of the surface resistance at the peak of the $Q_{0}(H)$ curve, exhibits lowest values after a single HF rinse followed by an upward trend with further HF rinses. The value of $b$ is affected by the residual resistance and the interplay between the MFQS and LFQS and thus cannot be used to reflect on the depth of LFQS.

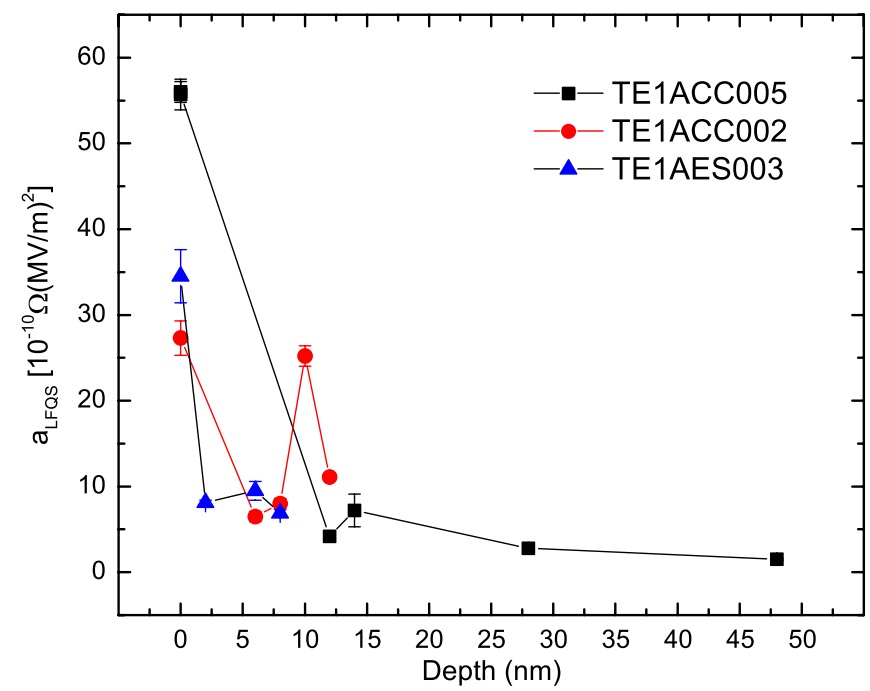

FIG. 7. LFQS fit results for the slope-defining parameter $a$ as a function of material removal depth. Error bars describe standard errors of the fit.

\section{Medium field $Q$ slope}

Medium field $Q$ slope (MFQS) is a decrease in $Q_{0}$ with field over the peak magnetic field range of about 20-80 mT. Several mechanisms have been proposed to explain the effect. One of the mechanisms is based on the presence of normal conducting niobium hydrides, which would produce a linear dependence of extra surface resistance on $H$ as found for the case of $Q$ disease [20]. Halbritter [21] proposed an additional term in the surface resistance coming from hysteretic losses caused by Josephson fluxons entering niobium at "strong" links such as oxidized grain boundaries. Losses due to such fluxons are proportional to the magnetic field $H$ as well. Additionally, two separate quadratic in $H$ terms were suggested to come from thermal feedback due to the exponential dependence of the BCS surface resistance on temperature [17], and due to the nonlinear Meissner effect [22]. Weingarten [18] proposed small weakly superconducting defects at the surface of niobium as yet another possible source of the MFQS. Whichever model turns out to be correct should reflect the recently reported high magnetic field localization of the medium field losses [23].

For all of the models above, the following parametrization of the surface resistance is typically used for fitting the $Q_{0}(H)$ dependence in the medium field range:

$$
R_{s}=R_{0}\left[1+\gamma\left(\frac{H}{H_{c}}\right)^{2}\right]+R_{1}\left(\frac{H}{H_{c}}\right) .
$$

Previously reported MFQS studies on low beta cavities [24] show quadratic terms to be dominant. In high beta cavities, results differ between the labs with only the quadratic term in MFQS found at Saclay [25], while both 
TABLE IV. Parameters for the fits based on Eq. (2) of the medium field $Q$ slope in the bulk electropolished (EP) cavity (TE1ACC005), bulk EP + tumbling + light EP cavity (TE1ACC002), and a buffered chemically polished (BCP) cavity (TE1AES003).

\begin{tabular}{lrcc}
\hline \hline TE1ACC005 & $R_{0}(\mathrm{n} \Omega)$ & $R_{1}(\mathrm{n} \Omega)$ & $r^{2}$ \\
\hline EP not baked & 13.2 & 11.2 & 0.995 \\
$\mathrm{EP}+120^{\circ} \mathrm{C}$ & 7.2 & 18.8 & 0.995 \\
$\mathrm{HF}$ rinse $\times 1$ & 4.1 & 23.2 & 0.988 \\
$\mathrm{HF}$ rinse $\times 6$ & 11.9 & 16.3 & 0.988 \\
$\mathrm{HF}$ rinse $\times 7$ & 9.9 & 13.9 & 0.996 \\
$\mathrm{HF}$ rinse $\times 9$ & 9.1 & 12.7 & 0.999 \\
$\mathrm{HF}$ rinse $\times 14$ & 12.7 & 16.9 & 0.986 \\
$\mathrm{HF}$ rinse $\times 24$ & 15.0 & 9.6 & 0.995 \\
\hline $\mathrm{TE} 1 \mathrm{ACC} 002$ & & & \\
\hline $\mathrm{EP}+120^{\circ} \mathrm{C}$ & 6.2 & 13.8 & 0.982 \\
$\mathrm{HF}$ rinse $\times 1$ & 4.2 & 11.7 & 0.967 \\
$\mathrm{HF}$ rinse $\times 2$ & 7.7 & 17.6 & 0.981 \\
$\mathrm{HF}$ rinse $\times 3$ & 9.7 & 13.8 & 0.982 \\
$\mathrm{HF}$ rinse $\times 4$ & 8.5 & 9.7 & 0.984 \\
$\mathrm{HF}$ rinse $\times 5$ & 1.5 & 8.1 & $0.845^{\mathrm{a}}$ \\
$\mathrm{HF}$ rinse $\times 6$ & 10.1 & 10.6 & 0.989 \\
\hline TE1AES003 & & & \\
\hline $\mathrm{BCP}$ no bake & 11.9 & 36.2 & 0.989 \\
$\mathrm{BCP}+120^{\circ} \mathrm{C}$ & 4.0 & 41.8 & 0.998 \\
$\mathrm{HF}$ rinse $\times 1$ & 5.9 & 20.2 & 0.974 \\
$\mathrm{HF}$ rinse $\times 2$ & 4.8 & 20.0 & 0.992 \\
$\mathrm{HF}$ rinse $\times 3$ & 7.7 & 11.5 & 0.974 \\
$\mathrm{HF}$ rinse $\times 4$ & 8.5 & 15.1 & 0.993 \\
$\mathrm{HF}$ rinse $\times 5$ & & & 0.990 \\
\hline \hline $\mathrm{T}$ & & & \\
\hline
\end{tabular}

${ }^{\mathrm{a}}$ The fit is poor for this measurement due to the cable calibration problems during the rf test.

linear and quadratic dependences [15] were reported by other labs.

Using Eq. (3) for fits to our data we found that the best fit is provided with only a linear component present.

In Table IV values of the fit parameters providing the best fit (maximizing $r^{2}$ ) to our data are presented. Except for two cases when rf calibration problems were encountered during testing (footnoted in Table IV), the fits are excellent and describe well the medium field $Q$ slope. The change of the fit parameter $R_{1}$ with the approximate thickness of consumed niobium is shown in Fig. 8.

Two primary conclusions can be drawn from our data.

First, the slope-defining parameter $R_{1}$ is changing throughout the whole material removal sequence all the way down to $24 \mathrm{HF}$ rinses corresponding to about 36-48 nm of niobium. That means that, unlike LFQS, the MFQS origin in the cavities we used is residing within the larger thickness of several tens of nanometers.

Second, the fact that the quadratic term $\propto H^{2}$ in $R_{s}(H)$ is not required to fit our data indicates that suggested physical mechanisms based on the thermal feedback and nonlinear

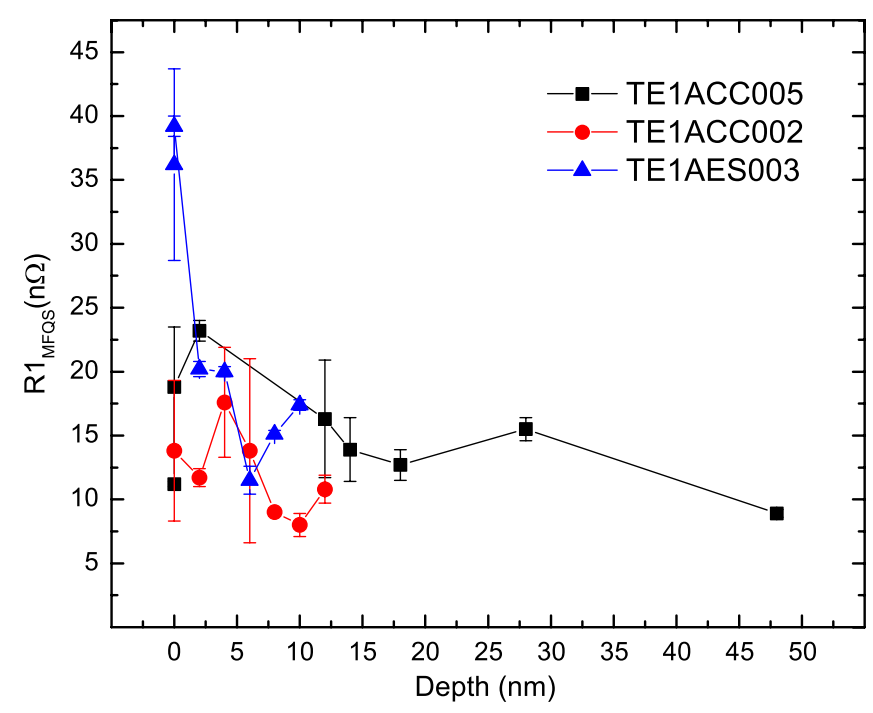

FIG. 8. MFQS fit results for the parameter $R_{1}$ with $\gamma=0$ for all fits as a function of material removal depth. Error bars describe standard errors of the fit.

Meissner effect are less probable. On the other hand, mechanisms leading to the linear term, among which are hydrides and oxidized strong links, are consistent with our observations.

\section{High field $Q$ slope}

The physical mechanism behind the high field $Q$ slope (HFQS) remains elusive despite the significant effort towards understanding this interesting phenomenon (see [26-28] for review). It was reported that a single HF rinse applied on mild baked cavities does not bring the HFQS back [26] but there is no data in the literature for multiple HF rinse cycles. Anodizing experiments $[4,5]$ showed that when about 20-30 $\mathrm{nm}$ of niobium are converted to oxide the HFQS reappears. This thickness corresponds to about 10-15 HF rinses in our experiments.

In our data we observe a gradual high field $Q$ slope reappearance in both TE1ACC002 (tumbled EP) and TE1ACC005 (EP) cavities. To demonstrate this point, a set of $\bar{R}_{s}=G / Q_{0}$ curves is shown for TE1ACC005 in Fig. 9. After six HF rinses $(\approx 9-12 \mathrm{~nm}$ of niobium consumed) the HFQS is present, but the onset field is higher than before $120^{\circ} \mathrm{C}$ bake. Subsequent HF rinses make the onset field lower and lower until after $24 \mathrm{HF}$ rinse cycles ( $\approx 36-48 \mathrm{~nm}$ of niobium consumed) $Q_{0}\left(B_{\text {peak }}\right)$ curve is essentially back to the prebaking shape. Therefore the required material removal to completely cancel the effect of mild baking on the HFQS is between 14 and $24 \mathrm{HF}$ rinses or in terms of thickness approximately 21-48 nanometers. It is in a reasonable agreement with the results of Eremeev [4] and Ciovati [5].

There is no accepted microstructural model for the origin of the HFQS. It is thus worth mentioning that recently we proposed a promising mechanism [29] for 


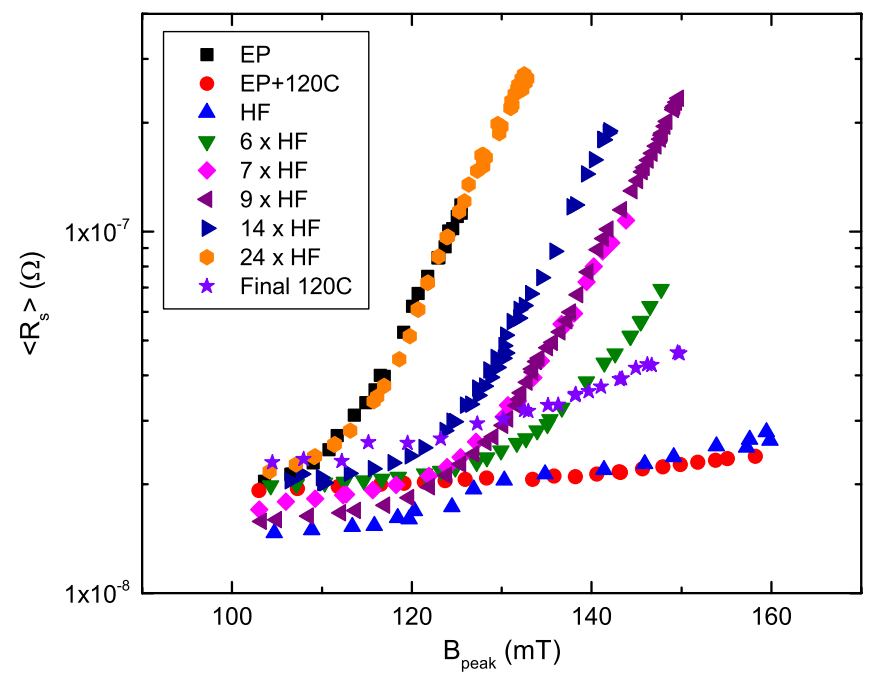

FIG. 9. Evolution of the high field $Q$ slope with multiple HF rinse cycles for the electropolished cavity TE1ACC005. Notice gradual emergence of the high field $Q$ slope as more and more niobium is consumed. Residual $Q$ slope after $120^{\circ} \mathrm{C}$ bake mimicking HFQS is due to field emission.

the HFQS based on the small niobium hydrides within the penetration depth. Within our model hydrides are superconducting by proximity effect up to the HFQS onset field. In further detail, we suggested that all the cavities have a significant hydrogen concentration in the near-surface layer. Accompanying such a concentration may be a layer of superabundant vacancy-hydrogen complexes with the concentrations of order a few $10^{-3}$ at. \%. Upon cooldown to $2 \mathrm{~K}$ interstitial near-surface hydrogen can form hydrides with vacancy-hydrogen complexes serving as nucleation centers. These small hydrides can be superconducting by proximity effect up to the critical field $H_{b} \propto 1 / d$, where $d$ is the hydride characteristic size. The mild baking effect may be explained by the dissociation and removal of Vac-H complexes thus providing much fewer sites for hydride nucleation. Our proximity model provides an excellent fit to the HFQS if a certain hydride size range is assumed. However, for the purpose of analyzing HF experiments where hydride size distribution and surface density may (and should within the model) change between the treatments, our model cannot provide a unique fit without additional input. Such input may be provided by either the observations of the predicted saturation of the HFQS, which would provide an upper limit on the hydride sizes, or by direct microscopic studies with TEM and SEM using in situ cold stage techniques to find such hydrides. Both projects are currently under way and results will be reported elsewhere.

\section{CONCLUSIONS}

We have studied the near-surface structure of mild baked SRF niobium cavities via material nanoremoval followed by rf testing. $Q_{0}\left(B_{\text {peak }}\right)$ curves, residual and BCS surface resistances, gap value, and electron mean-free path for different material removal thicknesses were obtained. A simple way to improve the quality factor based on hydrofluoric acid rinse was developed for practical applications. The low field $Q$ slope was found to be governed by the material structure within about 6 nanometers. Furthermore, the LFQS was found to be consistent in most cases with the inverse quadratic field dependence, which may be a signature of additional constant with field rf losses. Both medium and high field $Q$ slopes were found to be affected primarily by the material thickness of about 20-50 nanometers thick. MFQS was best fitted by the linear dependence of the surface resistance on field emphasizing possible roles of hydrides and strong links. HFQS was found to gradually reemerge with the thickness of the removed material and the depth of the mild baking effect on HFQS was found to be in agreement with previous studies.

\section{ACKNOWLEDGMENTS}

Authors would like to acknowledge Hasan Padamsee for suggesting the experiment and reading the manuscript, Charlie Cooper for tumbling one of the cavities, Dmitri Sergatskov for help with cavity tests, and Damon Bice and Allan Rowe for cavity processing. The work was partially supported by the DOE Office of Nuclear Physics. Fermilab is operated by Fermi Research Alliance, LLC under Contract No. DE-AC02-07CH11359 with the United States Department of Energy.

[1] H. Padamsee, RF Superconductivity: Volume II: Science, Technology and Applications (Wiley-VCH Verlag $\mathrm{GmbH}$ \& Co. KGaA, Weinheim, 2009).

[2] J. P. Charrier, B. Coadou, and B. Visentin, in Proceedings of the 6th European Particle Accelerator Conference, Stockholm, 1998 (IOP, London, 1998), pp 1885-1887 [http://www.jacow.org].

[3] P. Kneisel, in Proceedings of the 11th Workshop on RF Superconductivity (Los Alamos National Laboratory, Santa Fe, NM, 1999), TuP044 [http://www.jacow.org].

[4] G. Eremeev and H. Padamsee, Physica C 441, 62 (2006) [http://www.sciencedirect.com/science/article/pii/ S0921453406001523].

[5] G. Ciovati, P. Kneisel, and A. Gurevich, Phys. Rev. ST Accel. Beams 10, 062002 (2007).

[6] B. Visentin, J. P. Charrier, D. Roudier, Y. Gasser, A. Aspart, J. P. Poupeau, B. Coadou, and G. Monnereau, in Proceedings of the 11th Workshop on RF Superconductivity (Travemunde, Lubeck, Germany, 2003), MoP19 [http:// www.jacow.org].

[7] A. Agulyansky, Chemistry of Tantalum and Niobium Fluoride Compounds (Elsevier Science, New York, 2004).

[8] M. Grundner and J. Halbritter, J. Appl. Phys. 51, 397 (1980). 
[9] C. Antoine, A. Aspart, J. P. Charrier, H. Safa, and B. Visentin, in Proceedings of the 11th Workshop on RF Superconductivity (Ref. [3]), TuA008 [http://www .jacow.org].

[10] A. Romanenko and L. V. Goncharova, Supercond. Sci. Technol. 24, 105017 (2011).

[11] B. Aune et al., Phys. Rev. ST Accel. Beams 3, 092001 (2000).

[12] G. Ciovati, Report No. JLAB-TN-03-003.

[13] J. Halbritter, Technical Report No. FZK 3/70-6, Forschungszentrum Karslruhe, 1970.

[14] A. Grassellino, A. Romanenko, F. Barkov, and A. Suter, TFSRF' 2012 (2012) [http://www.jlab.org/ indico/conferenceDisplay.py?confId=22].

[15] G. Ciovati, ICFA Beam Dynamics Newsletter 39, 80 (2006) [http://www-bd.fnal.gov/icfabd/Newsletter39.pdf].

[16] G. Ciovati, J. Appl. Phys. 96, 1591 (2004).

[17] J. Halbritter, in Proceedings of the 38th Eloisitron Workshop, Erice, 1999 (World Science, Singapore, 2000), p. 59.

[18] W. Weingarten, Phys. Rev. ST Accel. Beams 14, 101002 (2011).

[19] V. Palmieri, in Proceedings of the 12th Workshop on RF Superconductivity (Cornell University, Ithaca, NY, 2005), p. 162 [http://www.jacow.org].

[20] J. Halbritter, P. Kneisel, and K. Saito, in Proceedings of the 6th Workshop on RF Superconductivity (Jefferson Lab,
Newport News, VA, 1993), pp. 617-627 [http://www .jacow.org].

[21] J. Halbritter, J. Appl. Phys. 97, 083904 (2005).

[22] A. Gurevich, Physica (Amsterdam) 441C, 38 (2006).

[23] A. Romanenko, J.P. Ozelis, A. Grassellino, and H. Padamsee, in Proceedings of IPAC'12 (New Orleans, LA, 2012), WEPPC116 [http://www.jacow.org].

[24] A. Grassellino, C. Beard, P. Kolb, R. Laxdal, D. Longuevergne, V. Zvyagintsev, and A. Romanenko, AIP Conf. Proc. 1352, 161 2010).

[25] B. Visentin, ICFA Beam Dynamics Newsletter 39, 94 (2006) [http://www-bd.fnal.gov/icfabd/Newsletter39 .pdf].

[26] B. Visentin, in Proceedings of the 11th Workshop on RF Superconductivity (Travemunde, Lubeck, Germany, 2003), TuO01 [http://www.jacow.org].

[27] G. Ciovati, in Proceedings of the 13th Workshop on RF Superconductivity (Beijing, China, 2007), p. 70 [http:// www.jacow.org].

[28] G. Ciovati, G. Myneni, F. Stevie, P. Maheshwari, and D. Griffis, Phys. Rev. ST Accel. Beams 13, 022002 (2010).

[29] A. Romanenko, F. Barkov, L. D. Cooley, and A. Grassellino, Supercond. Sci. Technol. (to be published) [http://arxiv.org/abs/1212.3384]. 